ment before and after simulated exercises. The study sample consisted of 45 undergraduate nursing students enrolled in a disaster management course. Content areas included types of disaster agents, nursing roles, and care of victims during a disaster event involving various lethal exposures and the use of appropriate personal protective equipment.

Results: Mean and standard deviation values for pre and post simulation were $77(4.04)$ and 86 (3.33) respectively. A one-sample $t$-test showed a statistically significant difference between pre and post simulation mean scores $(p<0.001)$. About $65 \%$ of students $(n=30)$ reported increased confidence in prioritizing care and performance of clinical skills after simulated experiences. Improved accuracy in the utilization of personal protective equipment also was reported.

Conclusion: Simulation experiences may enhance learning among undergraduate students in disaster management courses. During simulation, students acquire and improve clinical skills, critical thinking, and decisionmaking in a safe environment.

Keywords: disaster management; education; nurses; simulation; training

Prebosp Disast Med 2005;20(5):s167-s168

\section{Strengthening the Survival Chain in Disasters: Development of an Incident Medical Assistance Team Steven Trala, Raymond Scollin}

Fletcher Allen Health Care, Burlington, Vermont USA

Introduction: The Vermont Department of Health and Fletcher Allen Health Care have supported the development of an Incident Medical Assistance Team (IMAT) as a locally available, medically trained, incident response team. Within this framework, tactical medical response teams will be mobilized to incidents in which a field medical response unit or advanced medical practice team may augment the overall emergency response.

Objective: This presentation describes the development of an IMAT in Vermont.

Methods: Descriptive information was obtained from observations and records associated with this project.

Results: The IMAT was developed in four stages:

1. the initial idea was drafted, a field operations guide and equipment list were generated, and funding was applied for;

2. upon receipt of the first grant funds, initial equipment was purchased and recruitment of personnel began;

3. the field operations guide was revised, protocols were produced, team training took place, and additional equipment and supplies were purchased with continued grant funding; and

4. the IMAT was readied for deployment and systems and protocols underwent continuous re-evaluation.

Ultimately, three teams were developed with nine members each, including physicians, nurses, and emergency medical technicians at various certification levels. Each team is deployable within 90 minutes of notification.
The teams share a response vehicle, a trailer that stores a tent (inflatable in two minutes), a generator, 10 litters, ventilators, multiparameter monitor, various medical equipment, and medications. The next step is for the IMAT to beta-test its response at a mass casualty incident drill in autumn 2005.

Conclusion: By guaranteeing a physician is available in the field during a disaster, the IMAT provides an important link between emergency medical services and the emergency department and hospital trauma services, which may increase the chance of patient survival.

Keywords: disaster; emergency department; emergency medical services; incident medical assistance team; physician Prehosp Disast Med 2005;20(5):s168.

\section{Is Our Health Protected? A Connecticut Panel Tells} All

\section{Leon F. Vinci, ${ }^{1}$ William Blitz; ${ }^{2}$ Thomas Gecewicz; ${ }^{3}$ Rick} Fontana $a^{4}$

1. Chesprocott Health District, Cheshire, Connecticut USA

2. North Central District Health Department, Connecticut USA

3. Health Promotion Consultants, Lincoln, Nebraska USA

4. City of West Haven, Connecticut USA

Introduction: The terrorist attacks on 11 September 2001, and the terrorist anthrax letter attacks in October 2001 were critical in reshaping the US approach to emergency preparedness and response capability. To meet this challenge, Connecticut's public health community has changed its readiness capacity and infrastructure significantly. As a result, Connecticut's public health system has been improved and buttressed.

Objectives: This panel presentation will describe: (1) emergency readiness in the public health arena; (2) infrastructure change in Connecticut's public health emergency response system; and (3) how challenges in these processes were addressed in four Connecticut jurisdictions.

Other topics to be discussed include: (1) issues of encompassing health policy, legislation, funding, and government priorities; (2) the impact of the legislature, funding, state/local priorities, bioterrorism, and citizens on the process; (3) key legislation, funding, community priorities, and leadership challenges on this unique process; (4) community-wide priorities during this period and the unique involvement of a statewide professional health organization in the Connecticut experience; (5) planning and preparedness efforts for public health emergency response that have been taken throughout the state; (6) the strengthening of Connecticut's public health infrastructure; (7) competing interests and interagency relationships in accordance with this process; and (8) how advocacy, funding, legislation, and the threat of emergency/terrorism can propel public community priority and attention toward public health infrastructure enhancement. In addition, the alteration and reformation that the Connecticut public health system has experienced over the past three years will be reviewed from a community/region/state viewpoint in the context of addressing public health emergencies and threats. 
Methods: This presentation will be given by a panel of local community health experts in Connecticut.

Keywords: Connecticut; infrastructure; emergency readiness; emergency response; priorities; public health; terrorism Prehosp Disast Med 2005;20(5):s168-s169.

\section{Public Health Infrastructure Expansion: A Formula} for Success

Leon F. Vinci

Health Promotion Consultants, Lincoln, Nebraska USA

Introduction: Until recently, Nebraska ranked 51st in per capita spending on public health in the United States. As a result, the public health system (and emergency health preparedness) in Nebraska was fragmented, and without leadership. Today, the Nebraska public health system has grown dramatically, from $27 \%$, to nearly $100 \%$ coverage.

Objectives: This study describes the factors responsible for the recent public health infrastructure expansion in Nebraska.

Methods: In 2004, a survey of key informants in Nebraska was conducted regarding the factors responsible for the public health infrastructure expansion in the state. Key informants included public health leaders from statewide associations, government, local health agencies, academia, and advocacy groups.

Results: Survey participants reported that leadership, legislation, and funding were the most important factors in public health infrastructure enhancement. Another important factor was the need to for communities and government to develop an infrastructure for the response to public health emergencies.

Conclusion: Advocacy, legislation, funding, and the threat of an emergency or terrorism can facilitate the reformation of public health infrastructure.

Keywords: funding; leadership; legislation; public health; Nebraska; survey

Prebosp Disast Med 2005;20(5):s169.

Factors Impacting Public Health Emergency Preparedness in Local Communities: A Post-9/11 Survey

Leon F. Vinci

Health Promotion Consultants, Lincoln, Nebraska USA

Introduction: Connecticut's public health community experienced significant changes after the 2001 World Trade Center and Pentagon attacks and the 2001 US anthrax letter attacks.

Objectives: This presentation describes the new areas and services in Connecticut's public health emergency response system which have emerged since 11 September 2001, and the factors responsible for infrastructural change.

Methods: A retrospective analysis was performed of the steps, actions, and milestones in the governmental process as it related to the growth of public health preparedness in post-11 September 2001 Connecticut.
Information was obtained from surveying state and local health departments regarding the factors important for public health preparedness.

Results: Leadership, legislation, and funding were the most important factors in public health infrastructure enhancement in Connecticut since 11 September.

Conclusion: Advocacy, legislation, funding, and the threat of an emergency or terrorism can propel community attention and priorities toward public health infrastructure enhancement.

Keywords: advocacy; Connecticut; emergency response system; funding; leadership; legislation; public health; terrorism Prehosp Disast Med 2005;20(5):s169.

Pediatric Emergency Preparedness in General Hospital Emergency Departments in Connecticut James F. Wiley II; ${ }^{1}$ Asa Margolis, ${ }^{2}$ Kim Reich ${ }^{2}$

1. University of Connecticut School of Medicine, Farmington, Connecticut USA

2. Department of Epidemiology and Public Health, Yale University School of Medicine, New Haven, Connecticut USA

Introduction: The atrocity in Beslan, Russia in 2004 underscored the importance of emergency preparedness for children in disasters.

Objective: This study determines the level of pediatric preparedness and identifies resources needed in general hospital emergency departments (EDs) in Connecticut. Methods: This study was a retrospective survey of ED directors and nurse managers in 11 general hospitals in Connecticut. Data collected included: (1) demographic data; (2) content of hospital emergency operations plans (HEOPs); and (3) assessment of pediatric capability based on the Pediatric Preparedness for Disasters and Terrorism: National Consensus Conference in 2003, and the hospitals' rankings of the most needed pediatric resources.

Results: The average ED annual census was 40,954 $\pm 21,939$ visits with an average annual pediatric census of $6,533 \pm 5,168$ visits. Sixty-four percent of hospitals were located in suburban areas, and $36 \%$ provided residency training. Thirty percent had HEOPs that included pediatric guidelines, $20 \%$ had HEOPs that took into account the differences among children; $30 \%$ had HEOPs that contained a pediatric care resource; and $10 \%$ had HEOPs that provided for guardianship care/reunification of children separated from their parents. Seventy percent of hospitals typically lacked supplies for a pediatric victim surge of 100 children greater than their usual daily census. The top three resources needed were: (1) a pediatric treatment guide; (2) a pediatric field and hospital triage guide; and (3) a comprehensive web-based resource for pediatric management.

Conclusion: The ED leaders identified significant gaps in pediatric emergency preparedness at their hospitals, including pediatric components within their HEOPs to guide the management of pediatric victims of terrorist events and adequate equipment and medications to handle a surge of pediatric victims.

Keywords: emergency department; hospital emergency operations plan; pediatric emergency; pediatrics; preparedness Prebosp Disast Med 2005;20(5):s169. 